\title{
Peer navigation improves diagnostic follow-up after breast cancer screening among Korean American women: results of a randomized trial
}

\author{
Annette E. Maxwell • Angela M. Jo • \\ Catherine M. Crespi $\cdot$ Madhuri Sudan • \\ Roshan Bastani
}

Received: 5 January 2010/ Accepted: 19 July 2010/Published online: 31 July 2010

(c) The Author(s) 2010. This article is published with open access at Springerlink.com

\begin{abstract}
Objective To test an intervention to increase adherence to diagnostic follow-up tests among Asian American women. Methods Korean American women who were referred for a diagnostic follow-up test (mainly diagnostic mammograms) and who had missed their follow-up appointment were eligible to participate in the study. Women from two clinics $(n=176)$ were randomly allocated to a usual care control arm or a peer navigator intervention arm. A 20-min telephone survey was administered to women in both study arms six months after they were identified to assess demographic and socio-economic characteristics and the primary outcome, self-reported completion of the recommended follow-up exam.

Results Among women who completed the survey at 6month follow-up, self-reported completion of follow-up procedures was $97 \%$ in the intervention arm and $67 \%$ in the control arm $(p<0.001)$. Based on an intent-to-treat analysis of all women who were randomized and an assumption of no completion of follow-up exam for women with missing outcome data, self-reported completion of followup was $61 \%$ in the intervention arm and $46 \%$ in the usual care control arm $(p<0.069)$.

Conclusions Our results suggest that a peer navigator intervention to assist Korean American women to obtain
\end{abstract}

A. E. Maxwell $(\varangle) \cdot$ C. M. Crespi · M. Sudan · R. Bastani School of Public Health, University of California, Los Angeles and Jonsson Comprehensive Cancer Center, University of California, 650 Charles Young Drive South, A2-125 CHS, Box 956900, Los Angeles, CA 90095-6900, USA

e-mail: amaxwell@ucla.edu

A. M. Jo

David Geffen School of Medicine, University of California,

Los Angeles, CA, USA follow-up diagnostic tests after an abnormal breast cancer screening test is efficacious.

Keywords Mammography · Asian Americans · Medically uninsured $\cdot$ Health care disparities

\section{Introduction}

Until recently, the US Preventive Services Task Force recommended screening mammography, with or without clinical breast examination, every 1-2 years for women aged 40 and older based on evidence that screening significantly reduces mortality from breast cancer (http://www. ahrq.gov/clinic/3rduspstf/breastcancer/brcanrr.htm, accessed 20 October 2009).

As many as $15-20 \%$ of women who receive a screening mammogram and/or a clinical breast exam have abnormal or suboptimal/incomplete findings that require follow-up diagnostic tests [1-3]. However, many of these women never return to complete the recommended test. In most studies, fewer than $75 \%$ of patients receive follow-up tests [4] and in some underserved populations less than $50 \%$ of patients receive follow-up tests $[5,6]$.

Patient factors associated with incomplete and delayed follow-up after abnormal or suboptimal cancer screening tests include older age, low income and education level, nonwhite race, lack of social support, patients' lack of remembering the follow-up recommendation, concerns about cost, lost wages and transportation, and patients' fears [6-16]. Few studies have examined system factors such as inability to get appointments in a timely manner and provider barriers such as communication of the abnormal/incomplete finding and the need for follow-up tests $[4,17,18]$. To date, most studies on follow-up diagnostic tests are retrospective, descriptive 
studies [17]. A few prospective studies have shown that patient navigation is an effective strategy to increase adherence to diagnostic follow-up tests among Latino, African American and White women [19-24]. However, this strategy has not been tested among Asian American women.

This study was initiated in response to concerns from a clinic in Koreatown, Los Angeles, about missed diagnostic follow-up testing after breast cancer screening with abnormal or suboptimal findings among their predominantly immigrant Korean American patients. The purpose of this study was to test an intervention to assist Korean American women who obtain breast cancer screening through the Cancer Detection Program (CDP) and who require a followup test, usually a diagnostic mammogram. The intervention took place in the form of peer navigation, which included reminder phone calls, emotional support, translations, and other assistance to overcome barriers to follow-up that were identified during the initial phase of the study. The peer navigation strategy was chosen after discussions with clinic staff and informal interviews with Korean American patients and based on reports of the successes of patient navigation in other minority populations $[20,25]$.

\section{Methods}

\section{Overview and study design}

The study was conducted at two clinics in Koreatown that provided free screening through the CDP, a program funded by the Centers for Disease Control and Prevention and administered by the California Department of Public Health. Women 40 years of age and older with no or insufficient health insurance and income less than $200 \%$ of the poverty level are eligible to receive free annual breast cancer screening under this program. Korean American women were identified by CDP case managers based on their Korean first or last name. Only women who were referred for a diagnostic follow-up appointment, mostly based on the result of their clinical breast exam or screening mammogram, and who had missed their followup appointment were eligible to participate in the study because they were considered to be at highest risk for not completing their diagnostic procedures. Non-completion of the follow-up appointment was determined through a telephone call to the referral clinic. Almost all women in this study received a referral for a diagnostic mammogram and since the two participating clinics were not equipped to complete the diagnostic tests, all women were referred to an outside facility. One of the clinics referred all patients to one hospital, whereas the other clinic referred patients to the same hospital or a smaller facility, based on patient preference and proximity to residence.
The study used a post-randomization consent design, in which women were allocated to a study arm prior to obtaining informed consent [26]. Eligible women were randomly allocated to an intervention arm or a usual care arm of the study using a random number table at each clinic (Fig. 1). Usual care arm: Women received usual care according to the CDP protocol, consisting of up to two telephone calls by the CDP case manager and a registered letter, urging them to make an appointment for a follow-up exam. Intervention arm: Women were called within five days of their missed appointment and invited to participate in a study in which a Korean American peer navigator would assist them in obtaining their follow-up exam. Women who provided oral consent completed an intake form and received a peer navigator intervention in addition to usual care. Patients who declined received usual care but were analyzed as intervention group assignees. Six months after random allocation, all women were invited to participate in a telephone survey in Korean language. This was the first study contact for women in the usual care arm, and the interviewer obtained verbal consent prior to the interview.

Women in both arms of the study were also asked for permission to conduct a chart review to verify that all follow-up exams had been completed. Women in the intervention arm were usually asked by the peer navigator during a face-to-face encounter, while women in the control arm were asked after completion of the telephone survey. The study was approved by the Institutional Review Board of the University of California, Los Angeles and the Army Surgeon General's Human Subjects Research Review Board. The trial was registered at clinicalTrials.gov (NCT00742755).

\section{Intervention}

An English-Korean bilingual Korean American patient navigator was trained by one of the investigators (AMJ) to provide individually tailored assistance to the women in the intervention arm, including reminding women before an appointment, explaining the need for and the nature of the diagnostic follow-up exam, meeting women at the referral clinic, helping them to complete forms, and providing information and emotional support. Based on the Health Behavior Framework [27], the intervention was designed to increase knowledge and self-efficacy among women to complete the exam, and to assist women in overcoming barriers to completing the exam. The patient navigator was selected based on her ability to assist women to navigate the medical system and her ability to advocate for timely completion of the follow-up. After interviewing several potential candidates, we selected the wife of a local Korean pastor. She was in her late 40s, born in Korea, and had received a college education in the United States. 


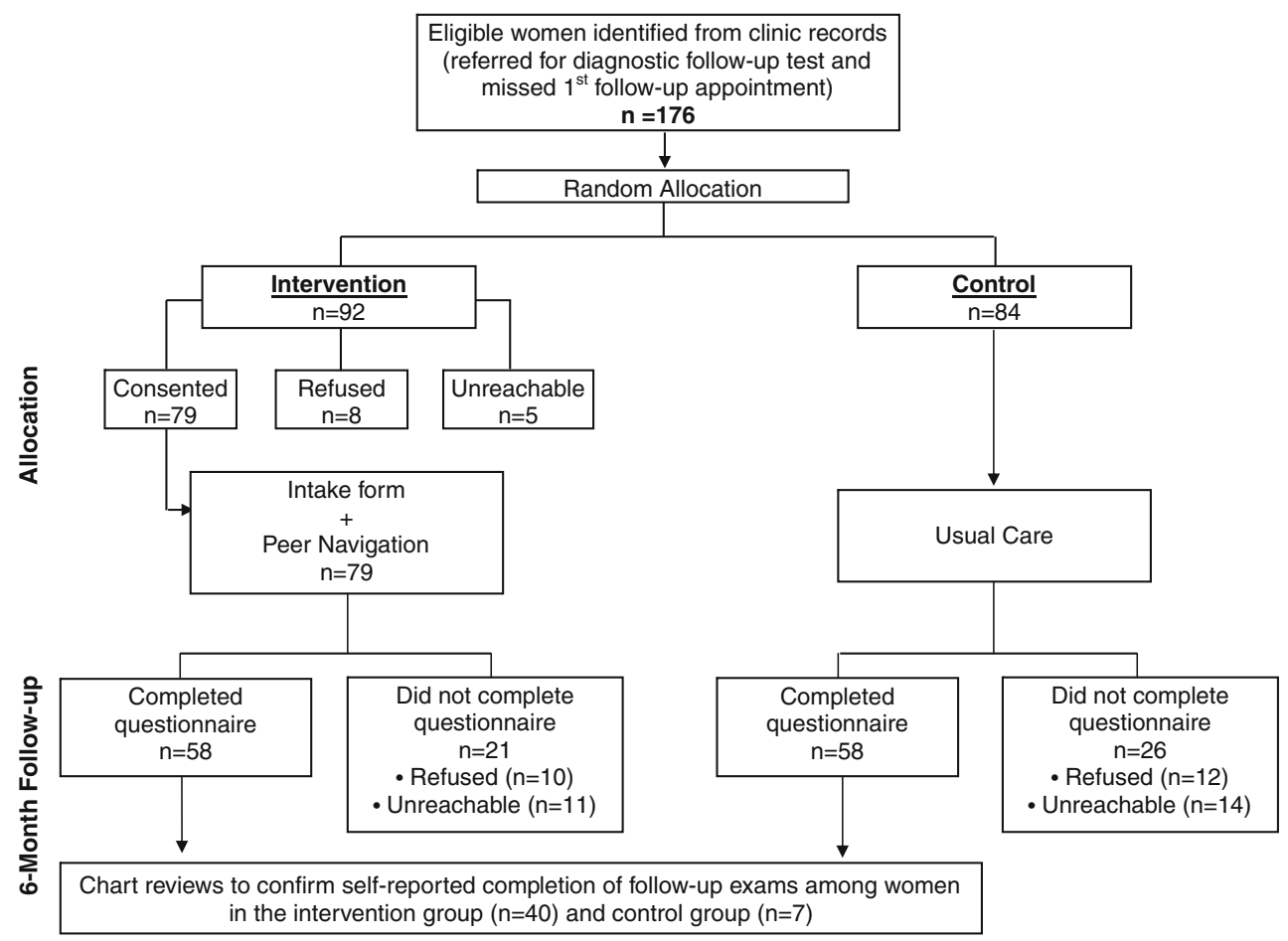

Fig. 1 Flow chart

Training of the peer navigator took place over the course of three half days and covered the following topics: breast health and cancer, focusing on prevention and early detection methods, screening abnormalities and follow-up diagnostics; pertinent key medical terms and concepts; CDP protocols and procedures; study protocol and forms involved with the study; information about the medical facilities involved, including information about the two study clinics and the typical referral centers; types of assistance to be offered to the women; and general etiquettes and safety precautions in working with strangers. Training took place face-to-face in an interactive discussion format in English and Korean language. The patient navigator was also given written materials and other resources from the American Cancer Society, the Komen Foundation, and the National Cancer Institute (i.e., videotapes, websites). Additionally, prior to any human subjects contact, she received IRB and HIPAA compliance trainings. One of the investigators (AMJ), a Korean American physician, met with the patient navigator once a week in person to monitor her activities and answer questions, with e-mail and phone contact in between as needed.

\section{Measures}

The patient navigator collected the following process measures: (1) She completed an intake form during her first telephone contact or face-to-face meeting with women assigned to the intervention arm that allowed her to tailor her assistance to each woman's individual needs. (2) In a log sheet, she noted all contacts and the type of assistance that she provided to each woman. Six months after women were identified, they completed a 20-min telephone survey that assessed demographic and socio-economic characteristics, including measures of acculturation, health insurance status, perceived health status, and the primary outcome: self-reported completion of the recommended follow-up exam. Subjects who completed the survey received \$20. Chart reviews were conducted for a total of 47 women who provided written consent for this part of the study.

Statistical analysis

We compared the intervention and controls groups on demographic and health-related characteristics using chisquare and Fisher exact tests. The number of services received and number of contacts with the peer navigator were compared between intervention arm completers and dropouts using the Mann-Whitney test. The primary outcome, self-reported completion of the recommended follow-up exam, was compared across the two arms using Fisher exact tests. We conducted this comparison among women who completed the study and we also conducted an intent-to-treat analysis, including all randomized subjects. The intent-to-treat analysis required an assumption about 
the percent of women with missing outcomes who had completed their follow-up exam. As a sensitivity analysis, we carried out the test for a range of assumptions, from $0 \%$ completion rate among women with missing outcomes to $60 \%$.

The sample size for the study was based on the numbers needed to achieve $80 \%$ power using a two-sided Fisher exact test with significance level of 0.05 assuming followup procedure adherence rates of 5 and $25 \%$ and using an analysis of subjects retained at follow-up.

\section{Results}

Between August 2005 and December 2007, we identified 176 eligible women from the CDP logs at the two participating clinics. We allocated 92 women to the intervention arm and 84 women to the usual care control arm of the study. We obtained consent to participate from 79/92 women in the intervention arm (86\%) and from 58/84 women in the usual care control arm (69\%). One of the reasons for the lower participation rate in the control arm is that women were first contacted six months after identification and a large number was unreachable at that time (14/ $84=17 \%$ ). Additionally, 12 women refused to participate. In comparison, in the intervention arm, in which women were contacted within five days after identification, only $5 \%(5 / 92)$ were unreachable. However, in the intervention arm, we lost an additional 21 women at 6-month follow-up. Thus, we completed 58 surveys in each arm of the study.

\section{Characteristics of study participants}

Table 1 reports sample characteristics, which are based on the 6-month telephone survey administered to both groups. Study participants were, on average, 52 years old. They were all foreign born and had lived in the United States for an average of 17 years. About three-quarters were married, and $80 \%$ reported an annual household income less than $\$ 30,000$. Most considered themselves more Korean than American (87\%), and the vast majority used Korean language with their friends, watched Korean TV and read Korean newspapers. Only $16 \%$ had health insurance. The low levels of income and health insurance in this sample are due to the eligibility criteria for the CDP.

Based on clinic records, most women had an abnormal or suboptimal mammogram $(76 \%)$ or an abnormal clinical breast exam (15\%) and had a referral for a diagnostic mammogram with or without ultrasound (97\%, see Table 2). This required an appointment at a larger clinic because both recruitment clinics only offered screening mammograms (two standard views) and neither was equipped to perform diagnostic mammograms (i.e., multiple or special views). Only one woman was referred for an ultrasound-guided core biopsy. Based on telephone survey data, only $10 \%$ of the women described their health status as excellent or very good, followed by good (24\%), fair (45\%), and poor $(21 \%)$. Most women stated that they were somewhat $(63 \%)$ or very comfortable $(31 \%)$ discussing health care with a provider. While most women stated that they were not at all or a little worried when they received the referral for a follow-up exam $(60 \%)$, a substantial minority was quite a bit or very much worried (40\%). No statistically significant differences between study arms were found on demographic or health-related variables that were assessed.

\section{Participants versus non-participants}

There were no statistically significant differences between women who consented to participate in the study and those who did not participate (refused or unreachable) with respect to age, clinic of recruitment, reason for diagnostic follow-up, and type of follow-up test needed.

\section{Completers versus dropouts, intervention arm only}

Of the 79 women in the intervention arm who consented to participate in the study, $58(73 \%)$ completed the 6-month telephone survey. There were no statistically significant differences between completers and dropouts with respect to age, clinic of recruitment, reason for diagnostic follow-up, and type of follow-up test needed (data not shown). Women who completed the 6-month survey and dropouts received a similar number of services from the peer navigator $(5.5 \pm 2.6$ vs. $4.9 \pm 2.8$, types of services are described below) and had a similar number of contacts (3.9 \pm 1.6 vs. $3.5 \pm 1.8$ ).

Process evaluation among women assigned to the peer navigation arm

The intake form that the peer navigator administered to women assigned to the intervention arm revealed that most women obtained the mammogram during which the potential abnormality was identified based on a doctor's recommendation or as part of their yearly routine screening (Table 3). However, a substantial proportion stated that they obtained the exam because they felt a lump (38\%) or because of pain or nipple discharge (17\%). Although most were treated politely at their last clinic visit and had their questions answered, large proportions stated that they had problems communicating with the doctor or the staff (74\%), that they found it difficult to ask questions (45\%), and that they had to wait too long to be seen $(33 \%)$. Although the costs of diagnostic follow-up exams are 
Table 1 Sample characteristics by study arm

\begin{tabular}{|c|c|c|c|c|c|c|c|}
\hline & \multicolumn{2}{|c|}{$\begin{array}{l}\text { Intervention } \\
(n=58)\end{array}$} & \multicolumn{2}{|c|}{$\begin{array}{l}\text { Control } \\
(n=58)\end{array}$} & \multicolumn{2}{|c|}{$\begin{array}{l}\text { Total } \\
(n=116)\end{array}$} & \multirow[t]{2}{*}{$p$-value ${ }^{\mathrm{a}}$} \\
\hline & $n$ & $\%$ & $n$ & $\%$ & $n$ & $\%$ & \\
\hline \multicolumn{8}{|l|}{ Age in years $(52 \pm 8,40-73)^{\mathrm{b}}$} \\
\hline $40-49$ & 24 & 41 & 17 & 35 & 41 & 39 & 0.794 \\
\hline $50-59$ & 22 & 38 & 21 & 44 & 43 & 41 & \\
\hline 60 or older & 12 & 21 & 10 & 21 & 22 & 21 & \\
\hline \multicolumn{8}{|l|}{ Marital status } \\
\hline Married & 41 & 77 & 39 & 75 & 80 & 76 & 0.777 \\
\hline Not married & 12 & 23 & 13 & 25 & 25 & 24 & \\
\hline \multicolumn{8}{|l|}{ Annual household income } \\
\hline$<\$ 10,000$ & 12 & 25 & 13 & 28 & 25 & 26 & 0.265 \\
\hline$\$ 10,000$ to $<\$ 20,000$ & 13 & 27 & 17 & 36 & 30 & 32 & \\
\hline$\$ 20,000$ to $<\$ 30,000$ & 14 & 29 & 6 & 13 & 20 & 21 & \\
\hline$\geq \$ 30,000$ & 9 & 19 & 11 & 23 & 20 & 21 & \\
\hline \multicolumn{8}{|l|}{ Education } \\
\hline$<$ High school & 8 & 15 & 7 & 13 & 15 & 14 & 0.400 \\
\hline High school/post-high school trade/technical school & 16 & 30 & 24 & 46 & 40 & 38 & \\
\hline $1-3$ years of college & 10 & 19 & 7 & 13 & 17 & 16 & \\
\hline$\geq 4$ years of college & 19 & 36 & 14 & 27 & 33 & 31 & \\
\hline \multicolumn{8}{|l|}{ Place of birth } \\
\hline Korea & 54 & 98 & 52 & 100 & 106 & 99 & 1.000 \\
\hline China & 1 & 2 & 0 & 0 & 1 & 1 & \\
\hline \multicolumn{8}{|l|}{ Years living in the US $(17 \pm 9,1-39)^{\mathrm{b}}$} \\
\hline$\leq 10$ & 20 & 36 & 18 & 35 & 38 & 36 & 0.239 \\
\hline $11-20$ & 13 & 24 & 19 & 37 & 32 & 30 & \\
\hline$\geq 21$ & 22 & 40 & 14 & 27 & 36 & 34 & \\
\hline \multicolumn{8}{|l|}{ Language used most of the time } \\
\hline Only Korean & 28 & 51 & 26 & 51 & 54 & 51 & 1.000 \\
\hline Mostly Korean & 23 & 42 & 22 & 43 & 45 & 42 & \\
\hline Only, mostly, half in English & 4 & 7 & 3 & 6 & 7 & 7 & \\
\hline \multicolumn{8}{|l|}{ Language in which read newspapers or magazines } \\
\hline Only Korean & 34 & 62 & 35 & 69 & 69 & 65 & 0.770 \\
\hline Mostly Korean & 17 & 31 & 13 & 25 & 30 & 28 & \\
\hline Only, mostly, half in English & 4 & 7 & 3 & 6 & 7 & 7 & \\
\hline \multicolumn{8}{|l|}{ Language in which watch television programs } \\
\hline Only Korean & 22 & 40 & 21 & 40 & 43 & 40 & 0.702 \\
\hline Mostly Korean & 19 & 35 & 21 & 40 & 40 & 37 & \\
\hline Only, mostly, half in English & 14 & 25 & 10 & 19 & 24 & 22 & \\
\hline \multicolumn{8}{|l|}{ Consider yourself more Korean vs. more American } \\
\hline More Korean & 47 & 87 & 44 & 86 & 91 & 87 & 0.909 \\
\hline Equal blend of both or more American & 7 & 13 & 7 & 14 & 14 & 13 & \\
\hline \multicolumn{8}{|l|}{ Have medical insurance } \\
\hline Yes & 9 & 16 & 8 & 15 & 17 & 16 & 0.856 \\
\hline No & 46 & 84 & 45 & 85 & 91 & 84 & \\
\hline
\end{tabular}

Frequencies do not sum to total sample due to missing responses

${ }^{\text {a }}$ Chi-square or Fisher exact tests

b Mean \pm standard deviation, range find a serious problem. About three-quarters of the women stated that they would like to receive a reminder about their follow-up appointment and one-quarter wanted more information about the recommended exam. 
Table 2 Health-related characteristics by study arm
Frequencies do not sum to total sample due to missing responses

${ }^{a}$ Chi-square or Fisher exact tests

\begin{tabular}{|c|c|c|c|c|c|c|c|}
\hline & \multicolumn{2}{|c|}{$\begin{array}{l}\text { Intervention } \\
(n=58)\end{array}$} & \multicolumn{2}{|c|}{$\begin{array}{l}\text { Control } \\
(n=58)\end{array}$} & \multicolumn{2}{|c|}{$\begin{array}{l}\text { Total } \\
(n=116)\end{array}$} & \multirow[t]{2}{*}{$p$-value ${ }^{\mathrm{a}}$} \\
\hline & $n$ & $\%$ & $n$ & $\%$ & $n$ & $\%$ & \\
\hline \multicolumn{8}{|l|}{ Reason for diagnostic follow-up } \\
\hline Abnormal mammogram & 34 & 76 & 31 & 78 & 65 & 76 & 0.833 \\
\hline Other & 11 & 24 & 9 & 23 & 20 & 24 & \\
\hline \multicolumn{8}{|l|}{ Type of follow-up test needed } \\
\hline Diagnostic mammogram and/or ultrasound & 44 & 79 & 38 & 76 & 82 & 77 & 0.786 \\
\hline Diagnostic mammogram 6-month follow-up & 10 & 18 & 11 & 22 & 21 & 20 & \\
\hline $\begin{array}{l}\text { Other (ultrasound guided core biopsy, } \\
\text { repeat mammogram, repeat CBE) }\end{array}$ & 2 & 4 & 1 & 2 & 3 & 3 & \\
\hline \multicolumn{8}{|l|}{ Health status } \\
\hline Poor & 14 & 25 & 9 & 17 & 23 & 21 & 0.285 \\
\hline Fair & 27 & 49 & 22 & 41 & 49 & 45 & \\
\hline Good & 10 & 18 & 16 & 30 & 26 & 24 & \\
\hline Very good/excellent & 4 & 7 & 7 & 13 & 11 & 10 & \\
\hline Have family history of cancer & 19 & 37 & 25 & 47 & 44 & 42 & 0.270 \\
\hline \multicolumn{8}{|l|}{ Comfort discussing health care with physician } \\
\hline Very comfortable & 17 & 33 & 14 & 28 & 31 & 31 & 0.623 \\
\hline Somewhat comfortable & 32 & 63 & 32 & 64 & 64 & 63 & \\
\hline Not comfortable at all & 2 & 4 & 4 & 8 & 6 & 6 & \\
\hline \multicolumn{8}{|c|}{ Worry about future health when doctor recommended exam } \\
\hline Very much worried & 9 & 16 & 5 & 10 & 14 & 13 & 0.699 \\
\hline Quite a bit worried & 15 & 27 & 14 & 27 & 29 & 27 & \\
\hline A little worried & 26 & 47 & 26 & 50 & 52 & 49 & \\
\hline Not at all worried & 5 & 9 & 7 & 13 & 12 & 11 & \\
\hline
\end{tabular}

The peer navigator provided the following services (number of women in parentheses): answered questions (69), made reminder calls (64), provided reassurance (57), provided in person help at the hospital (54), provided translation services (52), filled out forms (49), rescheduled appointment for follow-up test (42), gave directions to the hospital (23), provided transportation (2), made a home visit (1), provided other services (10). On average, the peer navigator provided $5.4( \pm 2.6)$ services per woman, and the average number of contacts per woman was $3.8 \pm 1.7$.

\section{Outcome evaluation}

Among women who completed the survey at 6-month follow-up, self-reported completion of follow-up procedures was $97 \%$ in the intervention arm and $67 \%$ in the control arm $(p<0.001$, Table 4$)$. Based on an intent-totreat analysis of all women who were randomized and an assumption of no completion of follow-up exam for women who refused participation, could not be contacted or dropped out, self-reported completion of follow-up was $61 \%$ in the intervention arm and $46 \%$ in the control arm $(p<0.069)$. It is probably too conservative to assume that all women who did not complete the 6-month survey did not complete their follow-up diagnostic exam. The true proportions completing diagnostic follow-up are likely to lie somewhere between the estimates derived from the two analysis approaches. If we assume that at least $5 \%$ of women in both arms of the study who did not complete the survey did complete diagnostic follow-up procedures, the difference in completion of follow-up procedures is statistically significant at $p<0.05$ (Table 4). Completion of follow-up procedures did not differ by the two clinics from which women were recruited nor by the two health care facilities to which they were referred.

Forty women in the intervention arm and 10 in the control arm gave permission to verify completion of follow-up appointments via chart reviews and we completed chart reviews for 47 women (three charts could not be found). Of the 40 charts reviewed for women in the intervention arm, all confirmed self-reported completion of the follow-up exam. In the control arm, five of seven charts confirmed self-reported completion. 
Table 3 Support and Barriers for Completing Follow-up Exam (Intervention Arm Only) $(n=58)$

\begin{tabular}{|c|c|c|}
\hline & $n$ & $\%$ \\
\hline \multicolumn{3}{|l|}{ Reasons for getting CBE/mammogram } \\
\hline Doctor recommended & 46 & 79 \\
\hline Get one every year & 33 & 57 \\
\hline Family/friends/TV suggested it & 24 & 41 \\
\hline Lump in breast & 22 & 38 \\
\hline Pain/nipple discharge & 10 & 17 \\
\hline \multicolumn{3}{|l|}{ Experience during last clinic visit } \\
\hline Treated politely & 54 & 93 \\
\hline Had problem communicating with doctor/staff & 43 & 74 \\
\hline Someone answered questions & 38 & 66 \\
\hline Found it difficult to ask questions & 26 & 45 \\
\hline Had to wait too long & 19 & 33 \\
\hline \multicolumn{3}{|l|}{ Factors supporting completion of follow-up exam } \\
\hline Understand recommended exam & 48 & 83 \\
\hline Have someone to talk/get help & 45 & 78 \\
\hline Can think of ways to work out problems & 44 & 76 \\
\hline $\begin{array}{l}\text { Able to ask relative/friend/neighbor } \\
\text { to accompany to clinic }\end{array}$ & 38 & 66 \\
\hline $\begin{array}{l}\text { Able to ask relative/friend/neighbor } \\
\text { for child/elder care during appointment }\end{array}$ & 32 & 55 \\
\hline Have a regular doctor & 12 & 21 \\
\hline \multicolumn{3}{|l|}{ Worries and concerns } \\
\hline Questions/worries about cost of exam & 54 & 93 \\
\hline Worried about finding serious problem from exam & 43 & 74 \\
\hline Worried about recommended exam or possible treatment & 40 & 69 \\
\hline Sometimes forget about medical appointments & 18 & 31 \\
\hline Have trouble scheduling follow-up exam & 17 & 29 \\
\hline Did not get follow-up exam b/c feel they don't need it & 10 & 17 \\
\hline Trouble getting transportation to clinic & 8 & 14 \\
\hline \multicolumn{3}{|l|}{ Needs } \\
\hline Would like to be reminded about appointment & 45 & 78 \\
\hline Want more information about recommended exam & 14 & 24 \\
\hline
\end{tabular}

Table 4 Self-reported completion of follow-up exam by study arm

\begin{tabular}{|c|c|c|c|c|c|}
\hline \multirow[t]{2}{*}{ Approach } & \multicolumn{2}{|c|}{ Intervention } & \multicolumn{2}{|c|}{ Control } & \multirow[t]{2}{*}{$p$-value } \\
\hline & $n$ & $\%$ & $n$ & $\%$ & \\
\hline Study completers & $56 / 58$ & 97 & $39 / 58$ & 67 & $<0.001$ \\
\hline \multicolumn{6}{|l|}{ Intent-to-treat analysis } \\
\hline Assumption of "no follow-up" for study non-completers & $56 / 92$ & 61 & $39 / 84$ & 46 & 0.069 \\
\hline Assumption of " $5 \%$ follow-up" for study non-completers & $58 / 92$ & 63 & $40 / 84$ & 48 & 0.049 \\
\hline Assumption of " $30 \%$ follow-up" for study non-completers & $66 / 92$ & 72 & $47 / 84$ & 56 & 0.040 \\
\hline Assumption of " $60 \%$ follow-up" for study non-completers & $76 / 92$ & 83 & $55 / 84$ & 65 & 0.010 \\
\hline
\end{tabular}

${ }^{\mathrm{a}}$ Fisher exact tests

\section{Discussion}

To our knowledge, this is the first study that tested in a randomized design the efficacy of a peer navigator intervention to increase diagnostic follow-up after breast cancer screening among Korean American women. We restricted our study to women who were thought to be at high risk for not completing recommended follow-up 
procedures-those who had already missed their first follow-up appointment-because the community clinics who may want to implement such a peer navigator intervention in the future have limited resources. Our findings were similar to studies conducted among non-Asian women [19, 21-23]. We found a clinically important and statistically significant increase in completion of follow-up tests in the intervention arm when compared to the usual care control arm of the study. Based on self-report, $67 \%$ of Korean American women in the usual care control arm received diagnostic follow-up tests (when compared to 64-78\% in the four studies cited previously) and $97 \%$ in the intervention arm (when compared to 78-96\% in the studies cited previously). Similar to another study that provided patient navigation to Korean American women based on their individual needs to help them obtain colorectal cancer screening [28], our study suggests that a culturally appropriate patient navigation intervention can reduce cancer disparities among Korean American women. There is also a growing body of literature that reports on the Patient Navigation Research Program, a cooperative effort of nine sites across the United States that are developing and testing peer navigation interventions among racial/ethnic minorities, individuals with lower socioeconomic status, and residents of rural areas [29, 30]. However, none of these studies target Asian Americans (http://crchd.cancer.gov/pnp/pnrpoverview.html, accessed 1/5/10). Our study supplements this literature by providing evidence of the efficacy of peer navigation among Korean American women.

This study used a post-randomization consent design, in which women were allocated to a study arm prior to obtaining informed consent, to avoid raising the expectation of getting help from a peer navigator among women who subsequently might be randomized to the usual care control arm. Other studies that tested patient navigation interventions have used similar study designs [22, 31] or have compared outcomes pre- and post-intervention [23]. We are reporting both completion rates as observed and completion rates based on intent-to-treat analysis. For the intent-to-treat analysis, we have presented estimates under a range of assumptions about completion rates because we believe that the traditional intent-to-treat analysis with imputation of "no follow-up" for all study non-completers is too conservative. In addition, the refusal rate was unequal, with $20 \%$ total at intake and follow-up in the intervention arm compared to $14 \%$ in the usual care control arm. Thus, the assumption that all missing outcomes are non-adherent leads to an overly conservative estimate of effect size. A more realistic assumption may be that at least a proportion of women who did not complete the study completed follow-up tests. Table 4 shows how different assumptions yield different estimates for test completion.
All of the studies mentioned previously tested a patient navigation intervention that was tailored toward the needs of individual patients. Navigators assisted patients with health education counseling and appointments and navigation of the health care system, which required patient follow-up tracking. Most studies hired navigators that resembled the racial/ethnic characteristics of their minority patients to increase the likelihood that services were provided in a culturally appropriate way that was acceptable to patients. Patient navigation was provided either by a professional navigator with relevant educational background and prior experience in a medical health care setting [19, 23], by a trained peer navigator [22], or a combination of both depending on the patient's level of need [32]. In our study, peer navigation was provided by a Korean American woman who had immigrated to the US at age 16 and who had no prior training or experience in the health care sector. After training, she was able to assist Korean American patients with most of their needs and only very rarely did she have to consult the medically trained co-investigator. The services that the peer navigator provided in our study are similar to those described in other populations, with the exception of "filling out forms." We found that Korean American women do not feel comfortable filling out forms, even when they are well educated and when the forms are provided in Korean language.

The number of contacts between patient navigators and patients that has been reported in the literature ranged from 2 among predominantly African-American women in Atlanta, Georgia [22], to 6.5 among foreign-born nonEnglish-speaking Latinas in Los Angeles, California [21, 32]. Vourlekis et al. [32] speculate that their Los Angeles sample required more intensive peer navigation due to the problematic resource system operating in Los Angeles. In our study, the peer navigator had, on average, 3.8 contacts and $91 \%$ of the patients who consented to be in the intervention arm of the study received at least one service. The peer navigator assisted about $65 \%$ of all women with translations, which may account for some of the peer navigator's time. All women in our study had received their initial mammogram through the CDP, which also covers the costs for diagnostic follow-up tests. This, and the fact that all patients were referred to one of two hospitals for follow-up care, may have simplified the peer navigation process. Almost all services were provided via telephone or when the peer navigator met the patient at the hospital. The peer navigator made only one home visit to one patient and provided transportation to the hospital for two patients. This information will be valuable for planning future patient navigation programs for Korean American women and may also apply to other immigrant populations. 
Limitations

Although we had a rigorous study design in which women were randomized, the total number of participants was small, similar to some of the other studies that tested the efficacy of peer navigator interventions [19, 22]. This was due to the fact that we only recruited from two community clinics that provide screening to Korean American women and to the fact that we limited eligibility to women who had missed their first follow-up appointment. Due to the slow rate of recruitment, a single peer navigator delivered the intervention. This limits the generalizability of our findings. In addition, findings may be biased because only $63 \%$ of women in the intervention group and $69 \%$ of women in the control group completed the 6-month followup survey, and because women in the intervention group were informed about the study and consented at baseline, whereas those in the control group were first contacted at 6-month follow-up.

It was not possible to blind the interviewer who completed the survey at 6-month follow-up to the group assignment of participants because women in the usual care control arm needed to be consented prior to the survey, but the women in the intervention arm did not. This may have introduced some bias, although a structured questionnaire with closed ended questions was used to assess the outcome among all women. To reduce social desirability bias, the study staff who conducted follow-up interviews was not involved in intervention activities.

In the original study proposal, we had planned to assess the outcome for all women from their charts, but with the implementation of the Health Insurance Portability and Accountability Act (HIPAA) Privacy Rule, this was no longer feasible. Participation in the chart review portion of the study was unequal. This is mainly because women in the usual care control arm had only a single telephone contact with study staff, after which they were mailed the HIPAA form and a prestamped and addressed envelope to return the form, while most women in the intervention arm had a face-to-face encounter, during which they were able to complete the HIPAA form and hand it back to the patient navigator. While we were not able to conduct sensitivity and specificity analyses due to small numbers, self-report of completion of follow-up exam agreed for the most part with chart data. This suggests that self-report is a valid outcome measure. With respect to the date of completion, however, we found large discrepancies (up to 6 months) between self-report and chart review in the small sample $(n=38)$ that completed both the survey and provided consent for chart review and where the date of the followup test was noted in the chart. Therefore, we did not consider self-reported time from identification of potential breast problem to completion of follow-up procedure as an outcome variable.

\section{Conclusion}

Completion rates of diagnostic follow-up procedures, based on self-report, were higher than we had expected in this sample of women who had already missed their first follow-up appointment. The majority of women in the control arm reported that they completed all follow-up procedures. However, completion rates were substantially higher in the intervention arm. These results suggest that a peer navigator intervention to assist Korean American women to obtain follow-up diagnostic tests after breast cancer screening is efficacious in this population.

Acknowledgments We would like to thank Ms. Hwang who served as peer navigator, the staff at the participating community clinics, and the women who participated in the study. Supported by US Army Medical Research and Materiel Command, DAMD17-03-1-0676. Dr. Crespi was also supported by NIH/NCI grant P30 CA16042 and Dr. Jo by NIH/NCI Grant R25 CA87949.

Open Access This article is distributed under the terms of the Creative Commons Attribution Noncommercial License which permits any noncommercial use, distribution, and reproduction in any medium, provided the original author(s) and source are credited.

\section{References}

1. Yabroff KR, Freedman A, Brown ML, Ballard-Barbash R, McNeel T, Taplin S (2007) Trends in abnormal cancer screening results in the United States of America. J Med Screen 14(2): $67-72$

2. Eheman CR, Benard VB, Blackman D, Lawson HW, Anderson C, Helsel W et al (2006) Breast cancer screening among lowincome or uninsured women: results from the National Breast and Cervical Cancer Early Detection Program, July 1995 to March 2002 (United States). Cancer Causes Control 17(1):29-38

3. Strzelczyk JJ, Dignan MB (2002) Disparities in adherence to recommended followup on screening mammography: interaction of sociodemographic factors. Ethn Dis 12(1):77-86

4. Yabroff KR, Washington KS, Leader A, Neilson E, Mandelblatt J (2003) Is the promise of cancer-screening programs being compromised? Quality of follow-up care after abnormal screening results. Med Care Res Rev 60(3):294-331

5. Mandelblatt J, Freeman H, Winczewski D, Cagney K, Williams S, Trowers R et al (1996) Implementation of a breast and cervical cancer screening program in a public hospital emergency department. Cancer Control Center of Harlem. Ann Emerg Med 28(5):493-498

6. Rojas M, Mandelblatt J, Cagney K, Kerner J, Freeman H (1996) Barriers to follow-up of abnormal screening mammograms among low-income minority women. Cancer Control Center of Harlem. Ethn Health 1(3):221-228

7. Peek ME, Han JH (2009) Compliance and self-reported barriers to follow-up of abnormal screening mammograms among women 
utilizing a county mobile mammography van. Health Care Women Int 30(10):857-870

8. Allen JD, Shelton RC, Harden E, Goldman RE (2008) Follow-up of abnormal screening mammograms among low-income ethnically diverse women: findings from a qualitative study. Patient Educ Couns 72(2):283-292

9. Press R, Carrasquillo O, Sciacca RR, Giardina EG (2008) Racial/ ethnic disparities in time to follow-up after an abnormal mammogram. J Womens Health 17(6):923-930

10. Yabroff KR, Breen N, Vernon SW, Meissner HI, Freedman AN, Ballard-Barbash R (2004) What factors are associated with diagnostic follow-up after abnormal mammograms? Findings from a US National Survey. Cancer Epidemiol Biomarkers Prev 13(5):723-732

11. Burack RC, Simon MS, Stano M, George J, Coombs J (2000) Follow-up among women with an abnormal mammogram in an HMO: is it complete, timely, and efficient? Am J Manag Care 6(10):1102-1113

12. McCarthy BD, Yood MU, Boohaker EA, Ward RE, Rebner M, Johnson CC (1996) Inadequate follow-up of abnormal mammograms. Am J Prev Med 12(4):282-288

13. McCarthy BD, Yood MU, Janz NK, Boohaker EA, Ward RE, Johnson CC (1996) Evaluation of factors potentially associated with inadequate follow-up of mammographic abnormalities. Cancer 77(10):2070-2076

14. Michielutte R, Diseker RA, Young LD, May WJ (1985) Noncompliance in screening follow-up among family planning clinic patients with cervical dysplasia. Prev Med 14(2):248-258

15. Chang SW, Kerlikowske K, Napoles-Springer A, Posner SF, Sickles EA, Perez-Stable EJ (1996) Racial differences in timeliness of follow-up after abnormal screening mammography. Cancer 78(7):1395-1402

16. Crane LA (1996) Social support and adherence behavior among women with abnormal Pap smears. J Cancer Educ 11(3):164-173

17. Wujcik D, Fair AM (2008) Barriers to diagnostic resolution after abnormal mammography: a review of the literature. Cancer Nurs 31(5):E16-E30

18. Bastani R, Yabroff KR, Myers RE, Glenn B (2004) Interventions to improve follow-up of abnormal findings in cancer screening. Cancer 101(5 suppl):1188-1200

19. Ferrante JM, Chen PH, Kim S (2008) The effect of patient navigation on time to diagnosis, anxiety, and satisfaction in urban minority women with abnormal mammograms: a randomized controlled trial. J Urban Health 85(1):114-124
20. Ell K, Padgett D, Vourlekis B, Nissly J, Pineda D, Sarabia O et al (2002) Abnormal mammogram follow-up: a pilot study women with low income. Cancer Pract 10(3):130-138

21. Ell K, Vourlekis B, Lee PJ, Xie B (2007) Patient navigation and case management following an abnormal mammogram: a randomized clinical trial. Prev Med 44(1):26-33

22. Crump SR, Shipp MP, McCray GG, Morris SJ, Okoli JA, Caplan LS et al (2008) Abnormal mammogram follow-up: do community lay health advocates make a difference? Health Promot Pract 9(2):140-148

23. Battaglia TA, Roloff K, Posner MA, Freund KM (2007) Improving follow-up to abnormal breast cancer screening in an urban population. A patient navigation intervention. Cancer 109(2 Suppl): 359-367

24. Freeman HP, Muth BJ, Kerner JF (1995) Expanding access to cancer screening and clinical follow-up among the medically underserved. Cancer Pract 3(1):19-30

25. Burhansstipanov L, Wound DB, Capelouto N, Goldfarb F, Harjo L, Hatathlie L et al (1998) Culturally relevant "navigator" patient support. The native sisters. Cancer Pract 6(3):191-194

26. Zelen M (1979) A new design for randomized clinical trials. N Engl J Med 300(22):1242-1245

27. Bastani R, Glenn BA, Taylor VM, Chen MS Jr, Nguyen TT, Stewart SL et al (2010) Integrating theory into community interventions to reduce liver cancer disparities: The health behavior framework. Prev Med 50(1-2):63-67

28. Ma GX, Shive S, Tan Y, Gao W, Rhee J, Park M et al (2009) Community-based colorectal cancer intervention in underserved Korean Americans. Cancer Epidemiol 33(5):381-386

29. Wells KJ, Battaglia TA, Dudley DJ, Garcia R, Greene A, Calhoun E et al (2008) Patient navigation: state of the art or is it science? Cancer 113(8):1999-2010

30. Freund KM, Battaglia TA, Calhoun E, Dudley DJ, Fiscella K, Paskett E et al (2008) National Cancer Institute Patient Navigation Research Program: methods, protocol, and measures. Cancer 113(12):3391-3399

31. Engelstad LP, Stewart S, Otero-Sabogal R, Leung MS, Davis PI, Pasick RJ (2005) The effectiveness of a community outreach intervention to improve follow-up among underserved women at highest risk for cervical cancer. Prev Med 41(3-4):741-748

32. Vourlekis B, Ell K, Padgett D (2005) Evidence-based assessment in case management to improve abnormal cancer screen followup. Health Soc Work 30(2):98-106 